\title{
Radiation from polarized electrons in oriented crystals at high energy
}

\author{
V. N. Baier and V. M. Katkov \\ Budker Institute of Nuclear Physics \\ 630090 Novosibirsk, Russia
}

November 10, 2018

\begin{abstract}
Radiation from high energy electrons in an oriented crystal can be considered in a frame of the quasiclassical operator method which appears to be a most satisfactory approach to the problem. Under some quite generic assumptions the general expression is derived for the probability of circularly polarized photon emission from the longitudinally polarized electron in oriented crystal. The particular mechanism of radiation depends on interrelation between the angle of incidence $\vartheta_{0}$ (angle between the momentum of initial electron and axis (plane) of crystal) and angle $\vartheta_{v} \equiv V_{0} / m$ ( $V_{0}$ is the scale of a potential of axis or a plane relative to which the angle $\vartheta_{0}$ is defined). When $\vartheta_{0} \ll \vartheta_{v}$ one has magnetic bremsstrahlung type of radiation (with corrections $\propto \vartheta_{0}^{2}$ which are due to inhomogeneous character of field in crystal). When $\vartheta_{0} \gg \vartheta_{v}$ one obtains the theory of coherent bremsstrahlung, while for $\vartheta_{0} \geq \vartheta_{v}$ one arrives to the modified theory of coherent bremsstrahlung. At high energy radiation in oriented crystals is strongly enhanced comparing with standard bremsstrahlung.
\end{abstract}




\section{Introduction}

The quasiclassical operator method developed by authors [1]- 3 is adequate for consideration of the electromagnetic processes at high energy. The probability of photon emission has a form (see [4], p.63, Eq.(2.27); the method is given also in $[5],[6])$

$$
d w=\frac{e^{2}}{(2 \pi)^{2}} \frac{d^{3} k}{\omega} \int d t_{2} \int d t_{1} R^{*}\left(t_{2}\right) R\left(t_{1}\right) \exp \left[-\frac{i \varepsilon}{\varepsilon^{\prime}}\left(k x\left(t_{2}\right)-k x\left(t_{1}\right)\right)\right],
$$

where $k^{\mu}=(\omega, \mathbf{k})$ is the 4-momentum of the emitted photon, $k^{2}=0, x^{\mu}(t)=$ $(t, \mathbf{r}(t)), t$ is the time, and $\mathbf{r}(t)$ is the particle location on a classical trajectory, $k x(t)=\omega t-\mathbf{k r}(t), \varepsilon^{\prime}=\varepsilon-\omega$, we employ units such that $\hbar=c=1$. The matrix

element $R(t)$ is defined by the structure of a current. For an electron (spin $1 / 2$ particle) one has

$$
\begin{aligned}
& R(t)=\frac{m}{\sqrt{\varepsilon \varepsilon^{\prime}}} \bar{u}_{s_{f}}\left(\mathbf{p}^{\prime}\right) \hat{e}^{*} u_{s_{i}}(\mathbf{p})=\varphi_{s_{f}}^{+}(A(t)+i \boldsymbol{\sigma} \mathbf{B}(t)) \varphi_{s_{i}}, \\
& A(t)=\frac{1}{2}\left(1+\frac{\varepsilon}{\varepsilon^{\prime}}\right) \mathbf{e}^{*} \boldsymbol{\vartheta}(t), \\
& \mathbf{B}(\mathbf{t})=\frac{\omega}{2 \varepsilon^{\prime}}\left(\mathbf{e}^{*} \times\left(\frac{\mathbf{n}}{\gamma}-\boldsymbol{\vartheta}(t)\right)\right),
\end{aligned}
$$

here $\mathbf{e}$ is the vector of the polarization of a photon (the Coulomb gauge is used), the four-component spinors $u_{s_{f}}, u_{s_{i}}$ and the two-component spinors $\varphi_{s_{f}}, \varphi_{s_{i}}$ describe the initial $\left(s_{i}\right)$ and final $\left(s_{f}\right)$ polarization of the electron, $\mathbf{v}=\mathbf{v}(\mathrm{t})$ is the electron velocity, $\boldsymbol{\vartheta}(t)=(\mathbf{v}-\mathbf{n}) \simeq \mathbf{v}_{\perp}(t), \mathbf{v}_{\perp}$ is the component of particle velocity perpendicular to the vector $\mathbf{n}=\mathbf{k} /|\mathbf{k}|, \gamma=\varepsilon / m$ is the Lorentz factor. The expressions in Eq.(1.2) are given for radiation of ultrarelativistic electrons, they are written down with relativistic accuracy (terms $\sim 1 / \gamma$ are neglected) and in the small angle approximation.

The important parameter $\chi$ characterizes the quantum effects in an external field, when $\chi \ll 1$ we are in the classical domain and with $\chi \geq 1$ we are already well inside the quantum domain

$$
\chi=\frac{|\mathbf{F}| \gamma}{F_{0}}, \quad \mathbf{F}=\mathbf{E}_{\perp}+(\mathbf{v} \times \mathbf{H}), \quad \mathbf{E}_{\perp}=\mathbf{E}-\mathbf{v}(\mathbf{v E}),
$$

where $\mathbf{E}(\mathbf{H})$ is an electric (magnetic) field, $F_{0}=m^{2} / e=\left(m^{2} c^{2} / e \hbar\right)$ is the quantum boundary (Schwinger) field: $H_{0}=4.41 \cdot 10^{13} \mathrm{Oe}, E_{0}=1.32 \cdot 10^{16} \mathrm{~V} / \mathrm{cm}$.

The quasiclassical operator method is applicable when $H \ll H_{0}, E \ll E_{0}$ and $\gamma \gg 1$.

The general expression for combination $R^{*}\left(t_{2}\right) R\left(t_{1}\right)=R_{2}^{*} R_{1}$ with all polarization taken into account is

$$
R_{2}^{*} R_{1} \equiv N_{21}\left(\boldsymbol{\zeta}_{i}, \boldsymbol{\zeta}_{f}, \mathbf{e}\right)=\frac{1}{4} \operatorname{Tr}\left[\left(1+\boldsymbol{\zeta}_{i} \boldsymbol{\sigma}\right)\left(A_{2}^{*}-i \boldsymbol{\sigma} \mathbf{B}_{2}^{*}\right)\left(1+\boldsymbol{\zeta}_{f} \boldsymbol{\sigma}\right)\left(A_{1}+i \boldsymbol{\sigma} \mathbf{B}_{1}\right)\right]
$$


where we use for the description of electron polarization the vector $\boldsymbol{\zeta}$ describing the polarization of the electron (in its rest frame), $\boldsymbol{\zeta}_{i}$ is the spin vector of initial

electron, $\boldsymbol{\zeta}_{f}$ is the spin vector of final electron. Summing over final spin states we have

$$
\sum_{\zeta_{f}} R_{2}^{*} R_{1}=A_{2}^{*} A_{1}+\mathbf{B}_{2}^{*} \mathbf{B}_{1}+i\left[A_{2}^{*}\left(\boldsymbol{\zeta}_{i} \mathbf{B}_{1}\right)-A_{1}\left(\boldsymbol{\zeta}_{i} \mathbf{B}_{2}^{*}\right)+\boldsymbol{\zeta}_{i}\left(\mathbf{B}_{2}^{*} \times \mathbf{B}_{1}\right)\right]
$$

where the two first terms describe the radiation of unpolarized electrons and the last terms is an addition dependent on the initial spin.

A combination of matrix elements $R_{2}^{*} R_{1}$ for spin-flip transition follows from Eq.(1.4) after substitution $\boldsymbol{\zeta}_{i}=\boldsymbol{\zeta}, \boldsymbol{\zeta}_{f}=-\boldsymbol{\zeta}$

$$
N_{21}(\boldsymbol{\zeta},-\boldsymbol{\zeta}, \mathbf{e})=\mathbf{B}_{2}^{*} \mathbf{B}_{1}-\left(\boldsymbol{\zeta} \mathbf{B}_{2}^{*}\right)\left(\boldsymbol{\zeta} \mathbf{B}_{1}\right)-i \boldsymbol{\zeta}\left(\mathbf{B}_{2}^{*} \times \mathbf{B}_{1}\right)
$$

It depends on the spin-flip amplitudes $\mathbf{B}_{1,2}$ only.

After summing in Eq.(1.5) over the polarization of emitted photon $\lambda$ we obtain for unpolarized electrons

$$
\sum_{\lambda, \zeta_{f}} R_{2}^{*} R_{1} \rightarrow \frac{1}{2 \varepsilon^{\prime 2}}\left[\frac{\omega^{2}}{\gamma^{2}}+\left(\varepsilon^{2}+\varepsilon^{\prime 2}\right) \boldsymbol{\vartheta}_{1} \boldsymbol{\vartheta}_{2}\right]
$$

For the longitudinally polarized initial electron and for circular polarization of emitted photon we have

$$
\sum_{\lambda, \zeta_{f}} R_{2}^{*} R_{1}=\frac{1}{2 \varepsilon^{\prime 2}}\left\{\frac{\omega^{2}}{\gamma^{2}}(1+\xi)+\left[(1+\xi) \varepsilon^{2}+(1-\xi) \varepsilon^{\prime 2}\right] \boldsymbol{\vartheta}_{1} \boldsymbol{\vartheta}_{2}\right\}
$$

where $\xi=\lambda \zeta, \lambda= \pm 1$ is the helicity of emitted photon, $\zeta= \pm 1$ is the helicity of the initial electron. In this expression we omit the terms which vanish after integration over angles of emitted photon.

Just for selected polarizations there are the strong effects in the radiation probability in high-energy limit: in the hard part of spectrum the longitudinally polarized initial electron emits mainly circularly polarized photon. This is a particular example of helicity transfer.

\section{General approach to radiation in oriented crys- tals}

The theory of high-energy electron radiation and electron-positron pair creation in oriented crystals was developed in [7]-[8], and given in [4. In these publications the radiation from unpolarized electrons was considered including the polarization density matrix of emitted photons. Since Eq.(1.8) has the same structure 
as Eq.(1.7), below we use systematically the methods of mentioned papers to obtain the characteristics of radiation from longitudinally polarized electron.

Let us remind that along with the parameter $\chi$ which characterizes the quantum properties of radiation there is another parameter

$$
\varrho=2 \gamma^{2}\left\langle(\Delta \mathbf{v})^{2}\right\rangle
$$

where $\left\langle(\Delta \mathbf{v})^{2}\right\rangle=\left\langle\mathbf{v}^{2}\right\rangle-\langle\mathbf{v}\rangle^{2}$ and $\langle\ldots\rangle$ denotes averaging over time. In the case $\varrho \ll 1$ the radiation is of a dipole nature and it is formed during the time of the order of the period of motion. In the case $\varrho \gg 1$ the radiation is of magnetic bremsstrahlung nature and it is emitted from a small part of the trajectory.

In a crystal the parameter $\varrho$ depends on the angle incidence $\vartheta_{0}$ which is the angle between an axis (a plane) of crystal and the momentum of a particle. If $\vartheta_{0} \leq \vartheta_{c}$ (where $\vartheta_{c} \equiv\left(2 V_{0} / \varepsilon\right)^{1 / 2}, V_{0}$ is the scale of continuous potential of an axis or a plane relative to which the angle $\vartheta_{0}$ is defined) electrons falling on a crystal are captured into channels or low above-barrier states, whereas for $\vartheta_{0} \gg \vartheta_{c}$ the incident particles move high above the barrier. In later case we can describe the motion using the approximation of the rectilinear trajectory, for which we find from Eq.(2.1) the following estimate

$$
\varrho\left(\vartheta_{0}\right)=\left(2 V_{0} / m \vartheta_{0}\right)^{2},
$$

For angles of incidence in the range $\vartheta_{0} \leq \vartheta_{c}$ the transverse (relative to an axis or a plane) velocity of particle is $v_{\perp} \sim \vartheta_{c}$ and the parameter obeys $\varrho \sim \varrho_{c}$ where

$$
\varrho_{c}=2 V_{0} \varepsilon / m^{2} .
$$

This means that side by side with the Lindhard angle $\vartheta_{c}$ the problem under consideration has another characteristic angle $\vartheta_{v}=V_{0} / m$ and $\varrho_{c}=\left(2 \vartheta_{v} / \vartheta_{c}\right)^{2}$.

We consider here the photon emission in a thin crystal when the condition $\varrho_{c} \gg 1$ is satisfied. In this case the extremely difficult task of averaging of Eq.(1.1) and Eqs.(1.4)-(1.8), derived for a given trajectory, over all possible trajectories of electrons in a crystal simplifies radically. In fact, if $\varrho_{c} \gg 1$ then in the range where trajectories are essentially non-rectilinear $\left(\vartheta_{0} \leq \vartheta_{c}, v_{\perp} \sim \vartheta_{c}\right)$ the mechanism of photon emission is of the magnetic bremsstrahlung nature and the characteristics of radiation can be expressed in terms of local parameters of motion. Then the averaging procedure can be carried out simply if one knows the distribution function in the transverse phase space $d N\left(\varrho, \mathbf{v}_{\perp}\right)$, which for a thin crystal is defined directly by the initial conditions of incidence of particle on a crystal. For a given angle of incidence $\vartheta_{0}$ we have $d N / N=d^{3} r F\left(\mathbf{r}, \vartheta_{0}\right) / V$, where $V$ is the volume of a crystal and $N$ is the total number of particles. In the axial case the function $F\left(\mathbf{r}, \vartheta_{0}\right)$ is of the form

$$
F_{a x}\left(\varrho, \vartheta_{0}\right)=\int \frac{d^{2} \varrho_{0}}{S\left(\varepsilon_{\perp}\left(\varrho_{0}\right)\right)} \vartheta\left(\left(\varepsilon_{\perp}\left(\varrho_{0}\right)-U(\varrho)\right),\right.
$$


where $U(\boldsymbol{\varrho})$ is the continuous axial potential dependent on the transverse coordinate $\varrho$ normalized so that $U(\boldsymbol{\varrho})=0$ at the boundary of a cell;

$$
S\left(\varepsilon_{\perp}\left(\varrho_{0}\right)\right)=\int \vartheta\left(\left(\varepsilon_{\perp}\left(\varrho_{0}\right)-U(\varrho)\right) d^{2} \varrho, \quad \varepsilon_{\perp}\left(\varrho_{0}\right)=\frac{\varepsilon \vartheta_{0}^{2}}{2}+U\left(\varrho_{0}\right)\right.
$$

where $\vartheta(x)$ is the Heaviside function: $\vartheta(x)=0$ if $x<0$ and $\vartheta(x)=1$ if $x>0$. For the planar case we have

$$
F_{p l}\left(x, \vartheta_{0}\right)=\int \frac{\vartheta\left(\varepsilon_{\perp 0}-U(x)\right) d x_{0}}{v\left(\varepsilon_{\perp 0}, x\right)}\left[\int \frac{\vartheta\left(\varepsilon_{\perp 0}-U(y)\right) d y}{v\left(\varepsilon_{\perp 0}, y\right)}\right]^{-1},
$$

where $U(x)$ is the continuous potential of plane dependent on the coordinate $x, v\left(\varepsilon_{\perp 0}, x\right)=\left[2\left(v\left(\varepsilon_{\perp 0}-U(x)\right) / \varepsilon\right]^{1 / 2}\right.$ is the transverse velocity of an electron, and $\varepsilon_{\perp 0}=\varepsilon \vartheta_{0}^{2} / 2+U\left(x_{0}\right)$. It should be noted that in the case when $\varepsilon_{\perp}\left(\varrho_{0}\right)>U_{0}$ (the above-barrier particles) the distribution Eq.(2.4) becomes uniform, whereas the distribution Eq.(2.6) becomes uniform only if $\varepsilon_{\perp 0} \gg U_{0}, U_{0}$ is the depth of potential well.

Substituting Eq.(1.8) into Eq.(1.1) we find after integration by parts of terms $\mathbf{n v}_{\mathbf{1 , 2}}\left(\mathbf{n v}_{\mathbf{1 , 2}} \rightarrow 1\right)$ the general expression for energy loss of radiating electron $\left(d E_{\xi}=\omega d w_{\xi}\right)$

$$
\begin{aligned}
& d E_{\xi}=-\frac{\alpha m^{2}}{8 \pi^{2}} \frac{d^{3} k}{\varepsilon \varepsilon^{\prime}} \int \frac{d^{3} r}{V} F\left(\mathbf{r}, \vartheta_{0}\right) \int e^{-i A}\left[\varphi_{1}(\xi)+\frac{1}{4} \varphi_{2}(\xi) \gamma^{2}\left(\mathbf{v}_{1}-\mathbf{v}_{2}\right)^{2}\right] d t_{1} d t_{2}, \\
& A=\frac{\omega \varepsilon}{2 \varepsilon^{\prime}} \int_{t_{1}}^{t_{2}}\left[\frac{1}{\gamma^{2}}+(\mathbf{n}-\mathbf{v}(t))^{2}\right] d t \\
& \varphi_{1}(\xi)=1+\xi \frac{\omega}{\varepsilon}, \quad \varphi_{2}(\xi)=(1+\xi) \frac{\varepsilon}{\varepsilon^{\prime}}+(1-\xi) \frac{\varepsilon^{\prime}}{\varepsilon} .
\end{aligned}
$$

where $\alpha=e^{2}=1 / 137$, the vector $\mathbf{n}$ is defined in Eq.(1.1), the helicity of emitted photon $\xi$ is defined in Eq.(1.8).

The circular polarization of radiation is defined by the Stoke's parameter $\xi^{(2)}$ :

$$
\xi^{(2)}=\Lambda(\boldsymbol{\zeta} \mathbf{v}), \quad \Lambda=\frac{d E_{+}-d E_{-}}{d E_{+}+d E_{-}}
$$

where the quantity $(\boldsymbol{\zeta} \mathbf{v})$ defines the longitudinal polarization of the initial electrons, $d E_{+}$and $d E_{-}$is the energy loss for $\xi=+1$ and $\xi=-1$ correspondingly. In the limiting case $\omega \ll \varepsilon$ one has $\varphi_{2}(\xi) \simeq 2(1+\xi \omega / \varepsilon)=2 \varphi_{1}(\xi)$. So the expression for the energy loss $d E_{\xi}$ contains the dependence on $\xi$ only as a common factor $\varphi_{1}(\xi)$ only. Substituting in Eq.(2.8) one obtains the universal result independent of a particular mechanism of radiation

$$
\xi^{(2)}=\frac{\omega}{\varepsilon}(\boldsymbol{\zeta} \mathbf{v})
$$


The periodic crystal potential $U(\mathbf{r})$ can be presented as the Fourier series (see e.g. 4, Sec.8 )

$$
U(\mathbf{r})=\sum_{\mathbf{q}} G(\mathbf{q}) e^{-i \mathbf{q} \mathbf{r}},
$$

where $\mathbf{q}=2 \pi\left(n_{1}, n_{2}, n_{3}\right) / l ; l$ is the lattice constant. The particle velocity can be presented in a form $\mathbf{v}(t)=\mathbf{v}_{0}+\Delta \mathbf{v}(t)$, where $\mathbf{v}_{0}$ is the average velocity. If $\vartheta_{0} \gg \vartheta_{c}$, we find $\Delta \mathbf{v}(t)$ using the rectilinear trajectory approximation

$$
\Delta \mathbf{v}(t)=-\frac{1}{\varepsilon} \sum \frac{G(\mathbf{q})}{q_{\|}} \mathbf{q}_{\perp} \exp \left[-i\left(q_{\|} t+\mathbf{q r}\right)\right]
$$

where $q_{\|}=\left(\mathbf{q} \mathbf{v}_{0}\right), \mathbf{q}_{\perp}=\mathbf{q}-\mathbf{v}_{0}\left(\mathbf{q} \mathbf{v}_{0}\right)$. If $\varrho_{c} \gg 1$, there is a range of angles $\vartheta_{0}$ which satisfies $\vartheta_{c} \ll \vartheta_{0} \ll \vartheta_{v}$, where both the magnetic bremsstrahlung description and the rectilinear trajectory approximation are valid. However, since the magnetic bremsstrahlung approach provides the same description for all angles $\vartheta_{0} \ll \vartheta_{v}$, the formula obtained in some range of $\vartheta_{0}$ remain valid up to $\vartheta_{0}=0$. It is known that in range $\vartheta_{0} \geq \vartheta_{v}$ the rectilinear trajectory approximation is valid as well for $\varrho_{c} \gg 1$. So, after integration in Eq.(2.7) over $\mathbf{u}=\mathbf{n}-\mathbf{v}_{0}\left(d^{3} k=\omega^{2} d \omega d \mathbf{u}\right)$ using Eq.(2.11) and passing to the variables $t, \tau: t_{1}=t-\tau, t_{2}=t+\tau$, we obtain after simple calculations the general expression for the intensity of radiation valid for any angle of incidence $\vartheta_{0}$

$$
\begin{aligned}
& d I_{\xi} \equiv \frac{d E_{\xi}}{d t}=\frac{i \alpha m^{2}}{4 \pi \varepsilon^{2}} \omega d \omega \int \frac{d^{3} r}{V} F\left(\mathbf{r}, \vartheta_{0}\right) \int \frac{d \tau}{\tau-i 0}\left[\varphi_{1}(\xi)-\varphi_{2}(\xi)\right. \\
& \left.\times\left(\sum_{\mathbf{q}} \frac{G(\mathbf{q})}{m q_{\|}} \mathbf{q}_{\perp} \sin \left(q_{\|} \tau\right) e^{-i \mathbf{q} \mathbf{r}}\right)^{2}\right] e^{-i A_{2}},
\end{aligned}
$$

where

$$
\begin{aligned}
& A_{2}=\frac{m^{2} \omega \tau}{\varepsilon \varepsilon^{\prime}}\left[1+\sum_{\mathbf{q}, \mathbf{q}^{\prime}} \frac{G(\mathbf{q}) G\left(\mathbf{q}^{\prime}\right)}{m^{2} q_{\|} q_{\|}^{\prime}}\left(\mathbf{q}_{\perp} \mathbf{q}_{\perp}^{\prime}\right) \Psi\left(q_{\|}, q_{\|}^{\prime}, \tau\right) \exp \left[-i\left(\mathbf{q}+\mathbf{q}^{\prime}\right) \mathbf{r}\right]\right] \\
& \Psi\left(q_{\|}, q_{\|}^{\prime}, \tau\right)=\frac{\sin \left(q_{\|}+q_{\|}^{\prime}\right) \tau}{\left(q_{\|}+q_{\|}^{\prime}\right) \tau}-\frac{\sin \left(q_{\|} \tau\right)}{q_{\|} \tau} \frac{\sin \left(q_{\|}^{\prime} \tau\right)}{q_{\|}^{\prime} \tau}
\end{aligned}
$$

\section{Radiation for $\vartheta_{0} \ll V_{0} / m$ (magnetic bremsstrahlung limit)}

The behavior of intensity $I$ Eq.(2.12) for various entry angles and energies is determined by the dependence on these parameters of the phase $A_{2}$ given Eq.(2.13). Here we consider the axial case for $\vartheta_{0} \ll V_{0} / m \equiv \vartheta_{v}$. The direction of crystal axis we take as $z$-axis of the coordinate system. The order of magnitude of the double sum in $A_{2}$ is $(G / m)^{2}\left(q_{\perp} / q_{\|}\right)^{2} \Psi\left(q_{\|}, q_{\|}^{\prime}, \tau\right)$. For the vector q lying in the 
plane $(x, y)$ we introduce notation $\mathbf{q}_{t}$, for such vectors one has $q_{z}=0$ and the quantities in Eq.(2.13) can be estimated in the following way:

$$
G(\mathbf{q}) \sim V_{0}, \quad q_{\perp} \sim 1 / a, \quad q_{\|} \sim \vartheta_{0} / a,
$$

where $a$ is the size of the region of action of the continuous potential. For all remaining vectors $q_{\perp} \sim q_{\|} \sim 1 / a$. Then the contribution to the sum of the terms with $q_{z} \neq 0$ will be $\sim\left(V_{0} / m\right)^{2} \Psi \leq\left(V_{0} / m\right)^{2}$. Since $\left(V_{0} / m\right)^{2} \ll 1$ this contribution can be neglected. Thus, we keep in the sum only terms with $\mathbf{q}_{t}$ for which its value is $\sim\left(V_{0} / m \vartheta_{0}\right)^{2} \Psi$. The large value of the phase $A_{2}$ leads to an exponential suppression of intensity $I$. Therefore the characteristic value of the variable $\tau$ in the integral Eq.(2.12) (which have the meaning of the formation time (length) of the process) will be adjusted in a such way that the large factor $\left(V_{0} / m \vartheta_{0}\right)^{2}$ will be compensated by the function $\Psi\left(q_{\|}, q_{\|}^{\prime}, \tau\right)$, i.e. for small entry angles the contribution gives region where $q_{\|} \tau \ll 1$. Expanding the phase $A_{2}$ correspondingly we find an approximate expression for $\vartheta_{0} \ll \vartheta_{v}$

$$
\begin{aligned}
& A_{2} \simeq \frac{m^{2} \omega \tau}{\varepsilon \varepsilon^{\prime}}\left\{1-\frac{\tau^{2}}{3} \sum_{\mathbf{q}_{t}, \mathbf{q}_{t}^{\prime}} G\left(\mathbf{q}_{t}\right) G\left(\mathbf{q}_{t}^{\prime}\right) \frac{\left(\mathbf{q}_{t} \mathbf{q}_{t}^{\prime}\right)}{m^{2}} \exp \left[-i\left(\mathbf{q}_{t}+\mathbf{q}_{t}^{\prime}\right) \boldsymbol{\varrho}\right]\right. \\
& \left.\times\left[1-\frac{\tau^{2}}{10}\left(\left(\mathbf{q}_{t} \boldsymbol{\nu}\right)^{2}+\left(\mathbf{q}_{t}^{\prime} \boldsymbol{\nu}\right)^{2}+\frac{2}{3}\left(\mathbf{q}_{t} \boldsymbol{\nu}\right)\left(\mathbf{q}_{t}^{\prime} \boldsymbol{\nu}\right)\right)\right]\right\},
\end{aligned}
$$

here $\boldsymbol{\nu}=\mathbf{v}_{0} / v_{0}$ is the direction of entry of the initial electron, $\boldsymbol{\varrho}=\mathbf{r}_{t}$. We cam rewrite Eq.(3.2) in the terms of the average potential of atomic string $U(\boldsymbol{\varrho})=\sum_{\mathbf{q}_{t}} G\left(\mathbf{q}_{t}\right) \exp \left(-i \mathbf{q}_{t} \boldsymbol{\varrho}\right)$ :

$$
A_{2}=\frac{m^{2} \omega \tau}{\varepsilon \varepsilon^{\prime}}\left\{1+\frac{\tau^{2}}{3} \mathbf{b}^{2} \tau^{2}+\frac{\tau^{4}}{15}\left[\left(\mathbf{b}(\boldsymbol{\nu} \nabla)^{2} \mathbf{b}\right)+\frac{1}{3}((\boldsymbol{\nu} \nabla) \mathbf{b})^{2}\right]\right\},
$$

where $\mathbf{b}=\boldsymbol{\nabla} U(\boldsymbol{\varrho}) / m, \boldsymbol{\nabla}=\partial / \partial \varrho$. For the pre-exponential factor in Eq.(2.12) we find

$$
[\ldots] \simeq \varphi_{1}(\xi)-\varphi_{2}(\xi) \tau^{2}\left[\mathbf{b}^{2}+\frac{\tau^{2}}{3}(\mathbf{b}(\nu \nabla) \mathbf{b})\right]
$$

Taking the integral over $\tau$ we obtain the spectral intensity for $\vartheta_{0} \ll V_{0} / m$.

$$
\begin{aligned}
& d I_{\xi}^{F}(\omega)=\frac{\alpha m^{2} \omega d \omega}{2 \sqrt{3} \pi \varepsilon^{2}} \int \frac{d^{2} \varrho}{s}\left\{\int \frac { d ^ { 2 } \varrho _ { 0 } } { s ( \varepsilon _ { \perp } ( \varrho _ { 0 } ) ) } \vartheta \left(\left(\varepsilon_{\perp}\left(\varrho_{0}\right)-U(\varrho)\right) R_{0}(\lambda)\right.\right. \\
& \left.-\frac{\left(\mathbf{b}(\boldsymbol{\nu} \boldsymbol{\nabla})^{2} \mathbf{b}\right)}{3 \mathbf{b}^{4}} R_{1}(\lambda)+\frac{\lambda}{30 \mathbf{b}^{4}}\left[((\boldsymbol{\nu} \nabla) \mathbf{b})^{2}+3\left(\mathbf{b}(\boldsymbol{\nu} \nabla)^{2} \mathbf{b}\right)\right] R_{2}(\lambda)\right\},
\end{aligned}
$$

where

$$
R_{0}(\lambda)=\varphi_{2}(\xi) K_{2 / 3}(\lambda)-\varphi_{1}(\xi) \int_{\lambda}^{\infty} K_{1 / 3}(y) d y
$$




$$
\begin{aligned}
& R_{1}(\lambda)=\varphi_{2}(\xi)\left(K_{2 / 3}(\lambda)-\frac{2}{3 \lambda} K_{1 / 3}(\lambda)\right), \\
& R_{2}(\lambda)=\varphi_{1}(\xi)\left(K_{1 / 3}(\lambda)-\frac{4}{3 \lambda} K_{2 / 3}(\lambda)\right) \\
& +\varphi_{2}(\xi)\left(\frac{4}{\lambda} K_{2 / 3}(\lambda)-\left(1+\frac{16}{9 \lambda^{2}}\right) K_{1 / 3}(\lambda)\right),
\end{aligned}
$$

here $\lambda=\frac{2 m^{2} \omega}{3 \varepsilon \varepsilon^{\prime}|\mathbf{b}|}, K_{\nu}(\sigma)$ is the modified Bessel function (McDonald's function). Since the expression for $d I^{F}$ is independent of $z$, it follows that $\int d^{3} r / V \rightarrow$ $\int d^{2} \varrho / s$, where $s$ is the transverse cross section area per axis. The term in Eq.(3.5) with $R_{0}(\lambda)$ represent the spectral intensity in the magnetic bremsstrahlung limit with the flux redistribution taken into account. The other terms are the correction proportional $\vartheta_{0}^{2}$.

If the potential $U(\varrho)$ can be considered as axially symmetric, we put $U=$ $U\left(\boldsymbol{\varrho}^{2}\right)$ and one can integrate over angles of vector $\varrho$. We obtain

$$
\begin{aligned}
& d I_{\xi}^{F}(\omega)=\frac{\alpha m^{2} \omega d \omega}{2 \sqrt{3} \pi \varepsilon^{2}} \int_{0}^{x_{0}} \frac{d x}{x_{0}}\left\{\int _ { 0 } ^ { x _ { 0 } } \frac { d y } { y _ { 0 } ( \varepsilon _ { \perp } ( y ) ) } \vartheta \left(\left(\varepsilon_{\perp}(y)-U(x)\right) R_{0}(\lambda)\right.\right. \\
& -\frac{1}{6}\left(\frac{m \vartheta_{0}}{V_{0}}\right)^{2}\left[\frac{x g^{\prime \prime}+2 g^{\prime}}{x g^{3}} R_{1}(\lambda)\right. \\
& \left.\left.-\frac{\lambda}{20 g^{4} x^{2}}\left(2 x^{2} g^{\prime 2}+g^{2}+14 g g^{\prime} x+6 x^{2} g g^{\prime \prime}\right) R_{2}(\lambda)\right]\right\},
\end{aligned}
$$

where we have adopted a new variable $x=\varrho^{2} / a_{s}^{2}, x \leq x_{0}, x_{0}^{-1}=\pi a_{s}^{2} d n_{a}=$ $\pi a_{s}^{2} / s, a_{s}$ is the effective screening radius of the potential of the string, $n_{a}$ is the density of atoms in a crystal, $d$ is the average distance between atoms of a chain forming the axis;

$$
\varepsilon_{\perp}(y)=\frac{\varepsilon \vartheta_{0}^{2}}{2}+U(y), \quad y_{0}\left(\varepsilon_{\perp}(y)\right)=\int_{0}^{\infty} \vartheta\left(\varepsilon_{\perp}(y)-U(x)\right) d x .
$$

The notation $U^{\prime}(x)=V_{0} g(x)$ is used in Eq.(3.7) and

$$
\lambda=\frac{m^{3} a_{s} \omega}{3 \varepsilon \varepsilon^{\prime} V_{0} g(x) \sqrt{x}}=\frac{u}{3 \chi_{s} g(x) \sqrt{x}}, \chi_{s}=\frac{V_{0} \varepsilon}{m^{3} a_{s}}, u=\frac{\omega}{\varepsilon^{\prime}}
$$

For specific calculation we use the following for the potential of axis:

$$
U(x)=V_{0}\left[\ln \left(1+\frac{1}{x+\eta}\right)-\ln \left(1+\frac{1}{x_{0}+\eta}\right)\right] .
$$

For estimates one can put $V_{0} \simeq Z e^{2} / d, \eta \simeq 2 u_{1}^{2} / a_{s}^{2}$, where $Z$ is the charge of the nucleus, $u_{1}$ is the amplitude of thermal vibrations, but actually the parameter 
of potential were determined by means of a fitting procedure using the potential Eq.(2.10) (table of parameters for different crystals is given in Sec.9 of [4]). For this potential

$$
g(x)=\frac{1}{x+\eta}-\frac{1}{x+\eta+1}=\frac{1}{(x+\eta)(x+\eta+1)}
$$

We shall assume, for the sake of simplicity, that the distribution is uniform over the transverse coordinates. This is true in the case of large angles of incidence $\vartheta_{0}>\vartheta_{c}$ and is approximately correct in the case of beams with a large angular spread $\Delta \vartheta_{0} \sim \vartheta_{c}$. In this case and for the assumptions $u \ll \chi_{s},\left(\chi_{s} / u\right)^{4 / 3} / \varrho\left(\vartheta_{0}\right) \ll 1$ we have

$$
\frac{d I_{\xi}^{M}}{\omega d \omega}=\frac{\alpha m^{2}}{6 \sqrt{3} \pi x_{0} \varepsilon^{2}} \Phi_{\xi}
$$

where

$$
\Phi_{\xi}=\Gamma\left(\frac{2}{3}\right)\left(\frac{6 \chi_{s}}{u}\right)^{2 / 3}\left[\varphi_{2}(u)\left(\ln \frac{18 \sqrt{3} \chi_{s}}{u}-\frac{\pi}{2 \sqrt{3}}-C-l_{1}(\eta)\right)-\frac{3}{2} \varphi_{1}(u)\right]
$$

here

$$
\begin{aligned}
& \varphi_{1}(u)=1+\frac{\xi u}{1+u}, \quad \varphi_{2}(u)=(1+\xi)(1+u)+\frac{1-\xi}{1+u}, \quad C=0.577216 \ldots \\
& l_{1}(\eta)=3.975 \beta^{2 / 3}\left(1+\frac{8}{15} \beta+\frac{7}{18} \beta^{2}\right)-\beta\left(\frac{3}{2}+\frac{9}{8} \beta+\frac{13}{14} \beta^{2}\right),
\end{aligned}
$$

where $\beta=\eta /(1+\eta)$. In derivation of Eq. (3.12) the integration over $x$ is carried out in the interval $(0, \infty)$. Since the main (logarithmic) contribution into the integral in Eq.(3.7]) comes from the interval $x \sim\left(\chi_{s} / u\right)^{2 / 3}$, it is clear that the asymptotic expression Eq.(3.12) is valid only is $\left(\chi_{s} / u\right)^{2 / 3}<x_{0}$.

At high energies $\left(\chi_{s} \gg 1\right)$ the expression for the intensity of radiation in the magnetic bremsstrahlung limit $I_{\xi}^{M}(\varepsilon)$ becomes

$$
I_{\xi}^{M}(\varepsilon) \simeq \frac{\alpha V_{0} g_{1} \varepsilon}{2 m x_{0} a_{s} \chi_{s}^{1 / 3}}\left[\left(1+\frac{11}{16} \xi\right)\left(\ln \chi_{s}+g_{0}\right)-\frac{231}{512} \xi\right],
$$

where

$$
\begin{aligned}
& g_{1}=\left(\frac{2}{3}\right)^{6} 6^{2 / 3} \Gamma\left(\frac{2}{3}\right) \simeq 0.3925 \\
& g_{0}=\frac{\pi}{2 \sqrt{3}}+\frac{5}{2} \ln 3+\ln 2-C-l_{1}(\eta) \simeq 0.6756-l_{1}(\eta) .
\end{aligned}
$$

The circular polarization of radiation is defined by Eq.(2.8), where we substitute $d E_{ \pm} \rightarrow d I_{ \pm}$. In the hard end of spectrum $\left(\varepsilon^{\prime} \ll \varepsilon\right) \Lambda \simeq 1$ since $d I_{+} \propto \varepsilon / \varepsilon^{\prime}$ 
and $d I_{-} \propto \varepsilon^{\prime} / \varepsilon$. In the limit $\chi_{s} \gg 1$ the radiation spectrum extends into hard part up to $u=\omega /(\varepsilon-\omega) \sim \chi_{s} a_{s} / u_{1}$, where under condition $|(\boldsymbol{\zeta} \mathbf{v})| \simeq 1$ the emitted photons have practically the complete circular polarization (the helicity coincide with electron helicity). For the integral radiation intensity (where the main contribution gives the region $\omega \sim \varepsilon-\omega)$ the circular polarization follows from Eq.(3.15):

$$
\Lambda=\frac{11}{16}\left(1-\frac{21}{32\left(\ln \chi_{s}+g_{0}\right)}\right) .
$$

Here the main term coincide with circular polarization of radiation of polarized electrons in external electromagnetic field, see Eq.(4.88) in 4 .

In Fig. 1 the spectral intensities of radiation $d I_{+}^{M} / d \omega, d I_{-}^{M} / d \omega$ and the spectral intensity of radiation of unpolarized electrons $d I^{M} / d \omega=d I_{+}^{M} / d \omega+d I_{-}^{M} / d \omega$ in tungsten crystal, axis $<111>$, temperature $T=293 K$ are plotted for energy $\varepsilon=250 \mathrm{GeV}$ (curves 1,2,3, respectively) and for energy $\varepsilon=1 \mathrm{TeV}$ (curves 4,5,6, respectively). It is seen that when $\omega / \varepsilon \rightarrow 1$ the intensity $d I_{+}^{M} / d \omega$ dominates $\left(d I_{+}^{M} / d \omega \gg d I_{-}^{M} / d \omega\right)$, while for $\omega / \varepsilon \ll 1$ the degree of polarization diminishes $\left(d I_{+}^{M} / d \omega \rightarrow d I_{-}^{M} / d \omega\right)$. It should be noted that Eq.(2.9) is fulfilled within accuracy better $10 \%$ for $\omega / \varepsilon \leq 0.2$. In Fig. 2 the circular polarization $\xi^{(2)}$ of radiation is plotted versus $\omega / \varepsilon$ for the same crystal. This curve is true for both energies: $\varepsilon=250 \mathrm{GeV}$ and $\varepsilon=1 \mathrm{TeV}$. Actually this means that it is valid for any energy in high-energy region. At $\omega / \varepsilon=0.8$ one has $\xi^{(2)}=0.94$ and at $\omega / \varepsilon=0.9$ one has $\xi^{(2)}=0.99$.

It is instructive to compare the obtained results with bremsstrahlung of polarized electrons in amorphous medium which one write as (see e.g. [6])

$$
\varepsilon \frac{d I_{\xi}^{C}}{d \omega}=\frac{\alpha m^{2} \varepsilon}{8 \pi \varepsilon_{e}}\left[(1+\xi)\left(1-\frac{2}{3} \frac{\varepsilon^{\prime}}{\varepsilon}\right)+\frac{\varepsilon^{\prime 2}}{\varepsilon^{2}}\left(1-\frac{\xi}{3}\right)+\frac{2}{9 L_{1}} \frac{\varepsilon^{\prime}}{\varepsilon}\left(1+\xi \frac{\omega}{\varepsilon}\right)\right],
$$

where $\varepsilon_{e}=m\left(8 \pi Z^{2} \alpha^{2} n_{a} \lambda_{c}^{3} L_{1}\right)^{-1}$, for notations see Eqs. (3.7) and (3.10), $\lambda_{c}=$ $1 / m$ is the electron Compton wavelength, $L_{1}=2\left(\ln 183 Z^{1 / 3}-f(Z \alpha)\right), f(z)=$ $\operatorname{Re}[\psi(1+i z)-\psi(1)], \psi(x)$ is the logarithmic derivative of the gamma function, the function $f(Z \alpha)$ gives the Coulomb corrections, for tungsten $L_{1}=6.99$, $\varepsilon_{e}=2.73 \mathrm{TeV}$. The dependence of the circular polarization $\xi^{(2)}$ on $\omega / \varepsilon$ for the intensity Eq.(3.18) is very close to shown in Fig.2. For the integral bremsstrahlung intensity Eq.3.18 one has $\Lambda=5 / 9$. However, as one can be seen from Fig.1, in the region $\omega \sim \varepsilon$ the magnitude of intensity in oriented crystal is around 10 times larger than given by Eq.(3.18) for both energies: $\varepsilon=250 \mathrm{GeV}$ and $\varepsilon=1 \mathrm{TeV}$. The value $r=\left(I^{M} / I^{C}\right)_{\max }=\left(L_{\text {rad }} / L_{c h}\right)_{\max }$ for different crystals is discussed in Sec.17.3 and is given in Table 17.1 in [4]. This value attains maximum value for light elements: in diamond for axis $<111>r=168$, in silicon for axis $<110>$ $r=81$.

It should be mentioned that for energies $\varepsilon \geq \varepsilon_{e}$ the process of hard photon emission $(\omega \sim \varepsilon)$ is affected by the multiple scattering (the LPM effect). At 
$\varepsilon \gg \varepsilon_{e}$ the suppression of spectral intensity of bremsstrahlung become essential for the whole spectrum [10, [11. On the contrary, the influence of multiple scattering on the photon emission in a oriented crystal is rather weak because the formation length in a oriented crystal is much shorter than in an amorphous medium (see Sec. 21 in [4).

\section{Modified theory of coherent bremsstrahlung}

The estimates of double sum in the phase $A_{2}$ made at the beginning of previous section: $\sim\left(\vartheta_{v} / \vartheta_{0}\right)^{2} \Psi$ remain valid also for $\vartheta_{0} \geq \vartheta_{v}$, except that now the factor in the double sum is $\left(\vartheta_{v} / \vartheta_{0}\right)^{2} \leq 1$, so that the values $\left|q_{\|} \tau\right| \sim 1$ contribute. We consider first the limiting case $\vartheta_{0} \gg \vartheta_{v}$, then this factor is small and $\exp \left(-i A_{2}\right)$ can be expanded accordingly. As a result Eq.(2.12) acquires the form

$$
\begin{aligned}
& d I_{\xi}^{c o h}(\omega)=-\frac{i \alpha m^{2} \omega d \omega}{4 \pi \varepsilon^{2}} \int_{-\infty}^{\infty} \frac{d \tau}{\tau-i 0} \exp \left(-i \frac{m^{2} \omega \tau}{\varepsilon \varepsilon^{\prime}}\right) \sum_{\mathbf{q}, \mathbf{q}^{\prime}} \frac{G(\mathbf{q}) G\left(\mathbf{q}^{\prime}\right)}{m^{2} q_{\|} q_{\|}^{\prime}}\left(\mathbf{q}_{\perp} \mathbf{q}_{\perp}^{\prime}\right) \\
& {\left[\varphi_{2}(\xi) \sin \left(q_{\|} \tau\right) \sin \left(q_{\|}^{\prime} \tau\right)+i \varphi_{1}(\xi) \frac{m^{2} \omega \tau}{\varepsilon \varepsilon^{\prime}} \Psi\left(q_{\|}, q_{\|}^{\prime}, \tau\right)\right] \int \frac{d^{3} r}{V} \exp \left[-i\left(\mathbf{q}+\mathbf{q}^{\prime}()^{\mathbf{4}}\right] 1\right)}
\end{aligned}
$$

The integration over coordinate $\mathbf{r}$ in Eq.(4.1) is elementary and gives $\delta_{\mathbf{q}+\mathbf{q}^{\prime}, 0}$, after which the sum over $\mathbf{q}^{\prime}$ and the integrals over $\tau$ are easily calculated by means of the theory of residues. Finally we obtain

$$
\begin{aligned}
& d I_{\xi}^{c o h}(\omega)=\frac{\alpha \omega d \omega}{8 \varepsilon^{2}} \sum_{\mathbf{q}}|G(\mathbf{q})|^{2} \frac{\mathbf{q}_{\perp}^{2}}{q_{\|}^{2}}\left[\varphi_{2}(\xi)-\varphi_{1}(\xi) \frac{2 m^{2} \omega}{\varepsilon \varepsilon^{\prime} q_{\|}^{2}}\left(\left|q_{\|}\right|-\frac{m^{2} \omega}{2 \varepsilon \varepsilon^{\prime}}\right)\right] \\
& \times \vartheta\left(\left|q_{\|}\right|-\frac{m^{2} \omega}{2 \varepsilon \varepsilon^{\prime}}\right) .
\end{aligned}
$$

The spectral distribution Eq. (4.2) coincide with the result of standard theory of coherent bremsstrahlung (CBS), see e.g. 9].

In the case $\chi_{s} \gg 1\left(\chi_{s}\right.$ is defined in Eq.(3.9) $)$, one can obtain from general expression Eq.(2.12) the expression for spectral intensity, the region of applicability of which is broader than that of standard CBS theory. For this purpose it is necessary to take into account that the phase $A_{2}$ Eq.(2.13) has for $q_{\|}+q_{\|}^{\prime} \neq 0$ terms of the order $\left(\vartheta_{v} / \vartheta_{0}\right)^{3} / \chi_{s}$ and $\left(\vartheta_{v} / \vartheta_{0}\right)^{4} / \chi_{s}^{2}$ which can be small even for $\vartheta_{0} \leq \vartheta_{v}$ if $\chi_{s} \gg 1$. Therefore, assuming that these contributions are small, we carry out the corresponding expansion of $\exp \left(-i A_{2}\right)$, while the term with $q_{\|}+q_{\|}^{\prime}=0$ in the double sum in $A_{2}$ will be retained in the exponent. As a result we obtain an expression which coincides in the form with Eq.(4.1) where we must make the substitution

$$
\exp \left(-i \frac{m^{2} \omega \tau}{\varepsilon \varepsilon^{\prime}}\right) \rightarrow \exp \left(-i \frac{m_{*}^{2} \omega \tau}{\varepsilon \varepsilon^{\prime}}\right), \quad m_{*}^{2}=m^{2}\left(1+\frac{\varrho}{2}\right) .
$$


Above the parameter $\varrho$ (Eq.(2.1) $)$ has the form

$$
\frac{\varrho}{2}=\frac{1}{m^{2}} \sum_{\mathbf{q}, \mathbf{q}^{\prime}} G(\mathbf{q}) G\left(\mathbf{q}^{\prime}\right) \frac{\mathbf{q}_{\perp} \mathbf{q}_{\perp}^{\prime}}{q_{\|} q_{\|}^{\prime}}\left[\delta_{q_{\|}+q_{\|}^{\prime}, 0}-\delta_{q_{\|}, 0} \delta_{q_{\|}^{\prime}, 0}\right]=\sum_{\mathbf{q}, q_{\|} \neq 0} \frac{|G(\mathbf{q})|^{2} \mathbf{q}_{\perp}^{2}}{m^{2} q_{\|}^{2}},
$$

and in the term $\frac{\sin \left(q_{\|}+q_{\|}^{\prime}\right) \tau}{\left(q_{\|}+q_{\|}^{\prime}\right) \tau}$ it is necessary to assume that $q_{\|}+q_{\|}^{\prime} \neq 0$. The remaining calculations are carried out in the same way as in the transition from Eq.(4.1) to Eq.(4.2). The final result can be presented in a form

$$
\begin{aligned}
& \frac{d I_{\xi}^{m c o h}(\omega)}{d \omega}=\frac{\alpha u}{8 \varepsilon} \sum_{\mathbf{q}}|G(\mathbf{q})|^{2} \frac{\mathbf{q}_{\perp}^{2}}{q_{\|}^{2}}\left[1+\xi+\frac{1-\xi}{(1+u)^{2}}\right. \\
& \left.-\frac{8[1+u(1+\xi)]}{(2+\varrho)(1+u)^{2}} \frac{u}{u_{0}}\left(1-\frac{u}{u_{0}}\right)\right] \vartheta\left(u_{0}-u\right),
\end{aligned}
$$

where

$$
u_{0}=\frac{4 \varepsilon q_{\|}}{m^{2}(2+\varrho)}, \quad u=\frac{\omega}{\varepsilon^{\prime}}
$$

Equation (4.5) is not more complicated than Eq.(4.2) but has a significantly broader range of applicability.

The spectral intensities Eqs.(4.2) and (4.5) can be much higher than the Bethe-Heitler bremsstrahlung intensity for small angles of incidence $\vartheta_{0}$ with respect to selected axis. For the case $\vartheta_{0} \ll 1$ the quantity $q_{\|}$can be represented as

$$
q_{\|} \simeq \frac{2 \pi}{d} n+\mathbf{q}_{\perp} \mathbf{v}_{0 . \perp}
$$

The main contribution to the sum in Eqs.(4.2) and (4.5) for small $\vartheta_{0}$ is given $\mathbf{q}$ with $n=0$, then

$$
q_{\|} \simeq\left(\frac{2 \pi}{f} k \cos \varphi+\frac{2 \pi}{h} l \sin \varphi\right) \vartheta_{0},
$$

where $f$ and $h$ are the characteristic periods of the potential in the transverse plane.

Let us consider the spectral intensity of radiation in the extreme limit, when the parameter $\lambda \equiv 2 \varepsilon\left|q_{\|}\right|_{\text {min }} / m^{2} \sim \varepsilon \vartheta_{0} / m^{2} a_{s} \gg 1$. In this case the maximum of intensity of coherent bremsstrahlung is attained at such values of $\vartheta_{0}$ where the standard theory of coherent bremsstrahlung becomes invalid. Bearing in mind that if $\lambda \gg 1$ and $\vartheta_{0} \sim V_{0} / m$, then $\chi_{s} \sim \lambda \gg 1$, we can conveniently use a modified theory of coherent bremsstrahlung.

The direction of transverse components of particle's velocity in Eq.(4.7) can be selected in a such way, that the spectral intensity described by Eq.(4.5) has a sharp maximum near the end of spectrum at $\omega=2 \varepsilon \lambda(2+2 \lambda+\varrho)^{-1}$ with relatively small (in terms of $\lambda^{-1}$ ) width $\Delta \omega \sim \varepsilon(1+\varrho / 2) / \lambda=m^{2}(1+\varrho / 2) / 2\left|q_{\|}\right|_{\text {min }}$ :

$$
\left(\frac{d I_{\xi}}{d \omega}\right)_{\max }=\frac{\alpha \varrho\left|q_{\|}\right|_{\min }}{4(2+\varrho)}\left(1+\xi+\frac{1-\xi}{\left(1+u_{m}\right)^{2}}\right), \quad u_{m}=\frac{2 \lambda}{2+\varrho} \text {. }
$$


It is seen that in the maximum of spectral distribution the radiation intensity with opposite helicity $(\xi=-1)$ is suppressed as $1 /\left(1+u_{m}\right)^{2}$. At $u>u_{m}$ one have to take into account the next harmonics of particle acceleration. In this region of spectrum the suppression of radiation intensity with opposite helicity is more strong, so the emitted photons have nearly complete circular polarization.

Comparing Eq.(4.9) with Eq.(3.18) for bremsstrahlung for $\varepsilon^{\prime} \ll \varepsilon$ we find that for the same circular polarization $\left(\xi^{(2)} \simeq(\boldsymbol{\zeta} \mathbf{v})\right)$ in the particular case $\varrho=1$ the magnitude of spectral intensity in Eq.(4.9) is about $\chi_{s}\left(\varepsilon_{e}\right)$ times larger than the spectral intensity in Eq.(3.18). For tungsten $\mathrm{W}$ one has $\chi_{s}\left(\varepsilon_{e}\right)=78$. Taking into account that $\Delta \omega \sim \varepsilon / \chi_{s}(\varepsilon)=\varepsilon_{e} / \chi_{s}\left(\varepsilon_{e}\right)$ we find that

$$
\Delta \omega\left(\frac{d I_{\xi}}{d \omega}\right)_{\max } \sim \frac{\varepsilon_{e}}{L_{\text {rad }}}, \quad \frac{d N_{\gamma}}{d t} \sim \frac{\varepsilon_{e}}{\varepsilon} \frac{1}{L_{\text {rad }}},
$$

where $N_{\gamma}$ is the number of emitted photons. So, it is seen from above analysis that under these conditions the considered mechanism of emission of photons with circular polarization is especially effective because there is gain both in monochromacity of radiation and total yield of polarized photons near hard end of spectrum.

\section{Conclusions}

It is shown above that at high energy the radiation from longitudinally polarized electrons in oriented crystals is circularly polarized and $\xi^{(2)} \rightarrow 1$ near the end of spectrum. This is true in magnetic bremsstrahlung limit $\vartheta_{0} \ll V_{0} / m$ as well as in coherent bremsstrahlung region $\vartheta_{0}>V_{0} / m$. This is particular case of helicity transfer.

It should be noted that in crossing channel: production of electron-positron pair with longitudinally polarized particles by the circularly polarized photon in an oriented crystal the same phenomenon of helicity transfer takes place in the case when the final particle takes away nearly all energy of the photon. The corresponding formulas can be obtained from given above using substitution rules. For processes in external electromagnetic field this item is discussed in Sec.3.3 of [4].

So, the oriented crystal is a very effective device for helicity transfer from an electron to photon and back from a photon to electron or positron. Near the end of spectrum this is nearly $100 \%$ effect.

\section{Acknowledgments}

The authors are indebted to the Russian Foundation for Basic Research supported in part this research by Grant 03-02-16154. 


\section{References}

[1] V. N. Baier, and V. M. Katkov, Phys. Lett.,A 25 (1967) 492.

[2] V. N. Baier, and V. M. Katkov, Sov.Phys.JETP 26 (1968) 854.

[3] V. N. Baier, and V. M. Katkov, Sov.Phys.JETP 28 (1969) 807.

[4] V. N. Baier, V. M. Katkov and V. M. Strakhovenko, Electromagnetic Processes at High Energies in Oriented Single Crystals, World Scientific Publishing Co, Singapore, 1998.

[5] V. B. Berestetskii, E. M. Lifshitz and L. P. Pitaevskii, Quantum Electrodynamics Pergamon Press, Oxford, 1982.

[6] V. N. Baier, V. M. Katkov and V. S. Fadin, Radiation from Relativistic Electrons (in Russian) Atomizdat, Moscow, 1973.

[7] V. N. Baier, V. M. Katkov and V. M. Strakhovenko, Sov.Phys.JETP 63 (1986) 467.

[8] V. N. Baier, V. M. Katkov and V. M. Strakhovenko, Sov.Phys.JETP 65 (1987) 686.

[9] M. L. Ter-Mikaelian, High Energy Electromagnetic Processes in Condensed Media, John Wiley \& Sons, 1972.

[10] V. N. Baier and V. M. Katkov, Phys.Rev. D 62 (2000) 036008.

[11] V. N. Baier and V. M. Katkov, "Concept of formation length in radiation theory" hep-ph/0309211, 2003; Preprint BINP 2003-58, Novosibirsk, 2003. 


\section{Figure captions}

- Fig.1 Spectral intensity of radiation $\varepsilon \frac{d I^{M}(\omega)}{d \omega}$ in tungsten crystal, axis $<111>, T=293 \mathrm{~K}$ in units $\alpha m^{2}$ vs $\omega / \varepsilon$. For the initial electron energy $\varepsilon=250 \mathrm{GeV}$ curve 1 is $d I_{-}^{M} / d \omega$, curve 2 is $d I_{+}^{M} / d \omega$, curve 3 is $d I_{+}^{M} / d \omega+$ $d I_{-}^{M} / d \omega$; and for the initial electron energy $\varepsilon=1 \mathrm{TeV}$ curve 4 is $d I_{-}^{M} / d \omega$, curve 5 is $d I_{+}^{M} / d \omega$, curve 6 is $d I_{+}^{M} / d \omega+d I_{-}^{M} / d \omega$.

- Fig.2 The circular polarization $\xi^{(2)}$ of radiation (for $(\boldsymbol{\zeta} \mathbf{v})=1$ ) vs $\omega / \varepsilon$ for the tungsten crystal, axis $<111>, T=293 \mathrm{~K}$. The curve is valid for both energies: $\varepsilon=250 \mathrm{GeV}$ and $\varepsilon=1 \mathrm{TeV}$. 


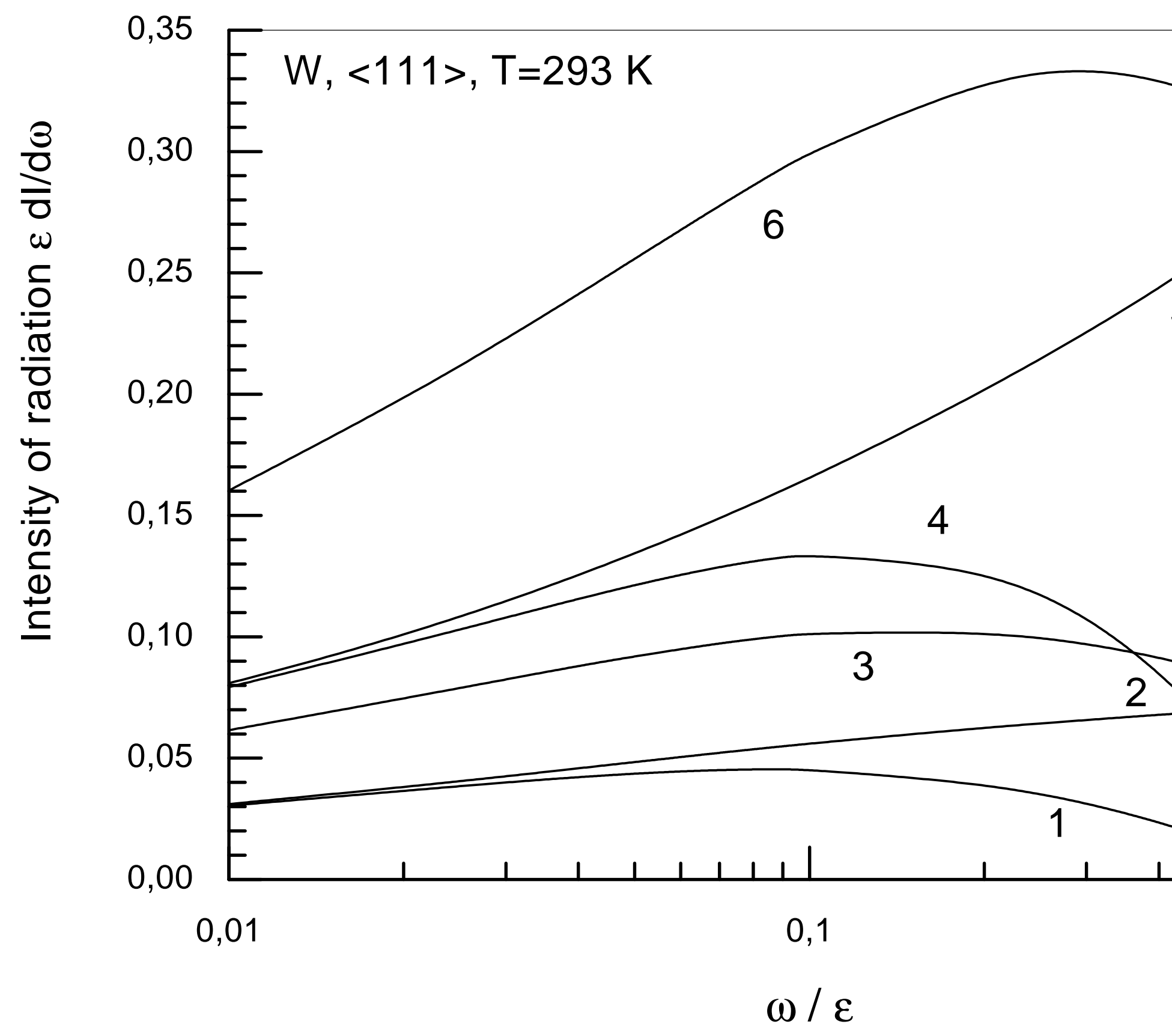




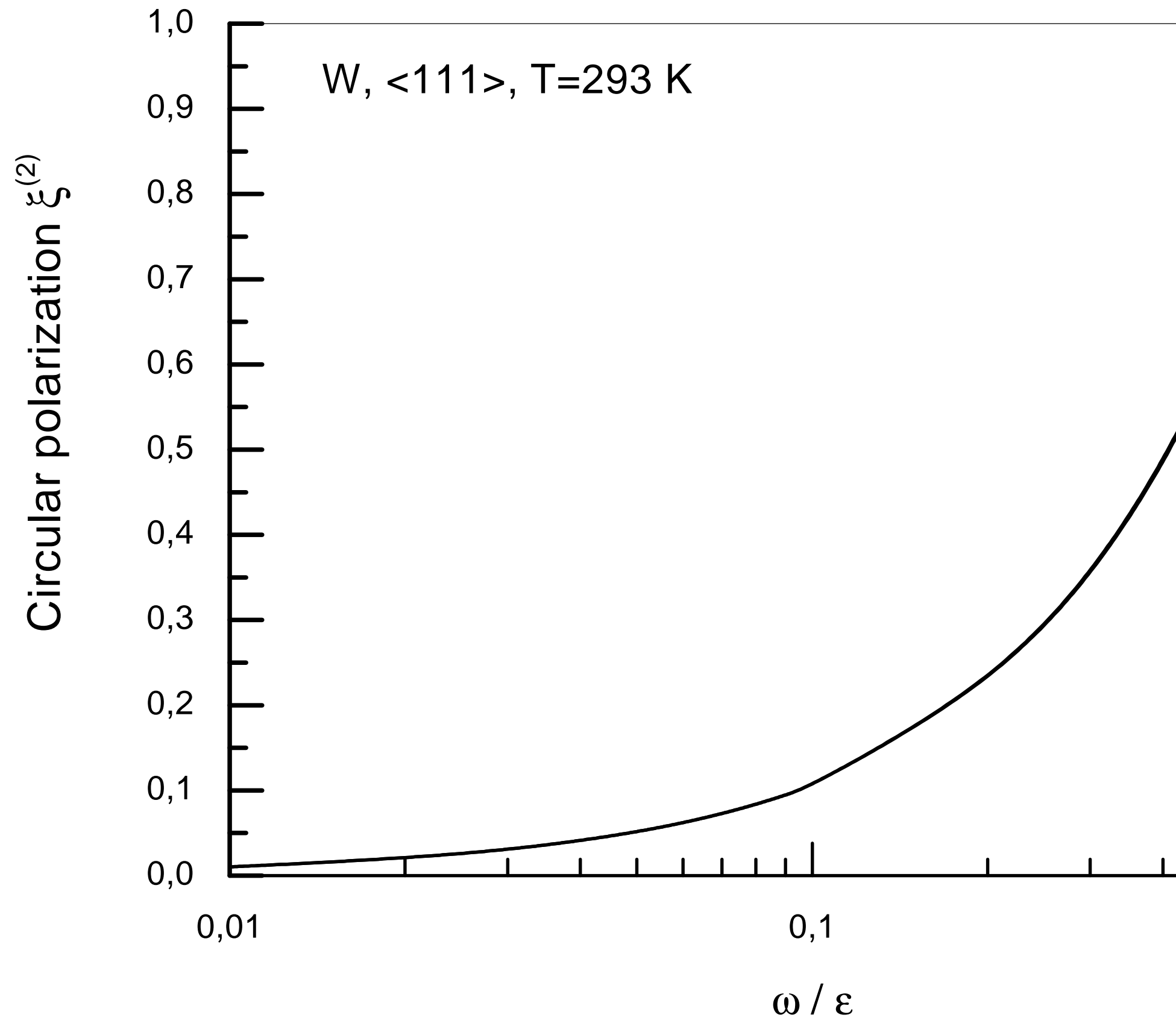

